\title{
Dissociated roles of the middle frontal gyri in the processing of Chinese characters
}

\author{
Chia-Li Liu ${ }^{\mathrm{a}}$, Chih-Wei Hue ${ }^{\mathrm{a}}$, Chien-Chung Chen ${ }^{\mathrm{a}}$, Kai-Hsiang Chuang ${ }^{\mathrm{b}}$, Keng-Chen Liang ${ }^{\mathrm{a}}$, \\ Yao-Hung Wang ${ }^{\mathrm{b}, \mathrm{c}}$, Chang-Wei Wu $\mathrm{W}^{\mathrm{b}}$ and Jyh-Horng Chen
}

\begin{abstract}
Departments of ${ }^{\mathrm{a} P s y c h o l o g y,}{ }^{\mathrm{b}}$ Electrical Engineering, Interdisciplinary MRI Laboratory, National Taiwan University and ${ }^{\mathrm{C}}$ Medical Imaging, National Taiwan University Hospital, Taipei, Taiwan, Republic of China

Correspondence and requests for reprints to Dr Chih-Wei Hue, PhD, Department of Psychology, National Taiwan University, Taipei I06, Taiwan, Republic of China

Tel: + 88623366 3086; fax: + 886223629909 ; e-mail: hue@ntu.edu.tw
\end{abstract}

Sponsorship: Grants from NHRI-EX92-9018EP to Professor J.H. Chen, NSC90-2413-H-002-024 to Professor K.C. Liang, and NSC91-273I-P-002-00I to NTU general funds.

Received 5 May 2006; accepted 7 June 2006

\begin{abstract}
The present study examined a hypothesis that the right middle frontal gyrus participates in processing orthography of Chinese characters, while the left middle frontal gyrus mediates access to phonology and semantics. Brain activation during three character tasks, which required processing orthography, phonology, or semantics of Chinese characters, respectively, was measured by functional magnetic resonance imaging. Comparable neural activity in the right middle frontal gyrus was observed in all three
\end{abstract}

character tasks that always demand orthographical processing. In contrast, the left middle frontal gyrus showed greater activation in the phonological and semantic tasks than in the orthographic task. These results suggest that the right and left middle frontal gyrus have dissociable functions in achieving Chinese character recognition. NeuroReport 17:1397-140I (C) 2006 Lippincott Williams \& Wilkins.

Keywords: blood oxygenation level-dependent, functional magnetic resonance imaging, language, orthography, phonology, semantics, word recognition

\section{Introduction}

Decoding written words is fundamental to reading. The mental processes underlying visual word recognition have been extensively studied, and various models have been proposed (for a review, see [1]). Different languages vary in their ways of mapping verbal words into script, making the decoding analysis even more complicated. The issue of whether different ways of representing words may affect word recognition in a specific written system remains to be resolved $[2,3]$.

This study aimed to dissociate functions of the left and right middle frontal gyrus in the processing of Chinese characters. This hypothesis is based on the following evidence. First, the left and right middle frontal gyri differ in their involvement in word recognition among various languages. Activation of the left side is routinely observed in literature of visual word recognition, irrespective of the type of written language being investigated, such as English [4], Korean [5], and Chinese [6]. In contrast, activation of the right side is reported in studies on Chinese characters, but to the best of our knowledge, has not been reported in studies on alphabetic words. Bilateral activation of the middle frontal gyrus was found in nearly all the studies on Chinese characters we reviewed [6-15] (Table 1, Fig. 1), irrespective of the diverse types of tasks, contrasts between very different tasks, such as semantic judgment minus fixation
$[9,14]$, and contrasts between naming different frequency characters [10,11]. The association of the right middle frontal gyrus, but not the left side, with the type of written language is striking, but its potential role in Chinese character recognition has not drawn much attention in the literature.

Second, functional dissociation between the left and right middle frontal gyrus has been suggested in working memory, which is strongly related to the mental operations involved. The left middle frontal gyrus is implicated in object working memory, whereas the right side is implicated in spatial working memory [16]. Therefore, we surmise that each side of the gyri is recruited, presumably with other neural regions, individually to fulfill different aspects of task demands, such as recognizing English words versus Chinese characters. Words can be regarded as symbolic objects and it is thus conceivable that recognizing words of different languages always engages the left middle frontal gyrus. Conversely, the logographic properties of Chinese characters possibly require additional neural analyses of the visuospatial features for compositional strokes [10,14], which the right middle frontal gyrus is capable of.

On the basis of the above evidence, we hypothesized that the right middle frontal gyrus subserves orthographic processing of Chinese characters, and the left counterpart mediates the conversion of orthography-to-phonology and 
Table I Summary of the tasks used and Talairach coordinates of activation in the left and right middle frontal gyri found in neuroimaging studies on Chinese character processing

\begin{tabular}{|c|c|c|c|c|}
\hline \multirow[b]{2}{*}{ Reference no. } & \multirow[b]{2}{*}{ Experimental task } & \multirow[b]{2}{*}{ Control task } & \multicolumn{2}{|c|}{ Talairach coordinates } \\
\hline & & & Left $(x, y, z)$ & Right $(x, y, z)$ \\
\hline [6] & Lexical judgment & Fixation & $-51,10,38$ & $53,27,28$ \\
\hline [7] & Reading character & Reading pinyin ${ }^{a}$ & $\mathrm{~N} / \mathrm{A}$ & $48,38,-8$ \\
\hline [8] & Word rehearsal & Picture-naming & $-52,35,30$ & $52,35,30$ \\
\hline [9] & Naming & Fixation & $-48,31,30$ & $34,47,24$ \\
\hline [10] & Naming LFC & Naming HFC & $-44,4,28$ & $42,8,24$ \\
\hline [III] & Naming HFC & Naming LFC & $-28,-5,48$ & $50,12,40$ \\
\hline [12] & Word generation & Fixation & $-47,19,26$ & $48,21,22$ \\
\hline [13] & Semantic judgment & Fixation & $-16,42,-17$ & $45,44,0$ \\
\hline & Homophone judgment & Fixation & $-43,13,27$ & $36,37,-14$ \\
\hline [14] & Semantic judgment & Fixation & $-54,12,36$ & $40,10,52$ \\
\hline [15] & Homophone judgment & Form judgment & $-44,10,34$ & $42,14,26$ \\
\hline
\end{tabular}

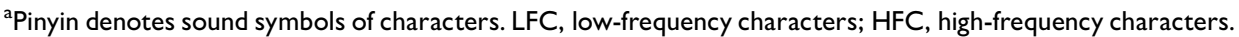

(a)
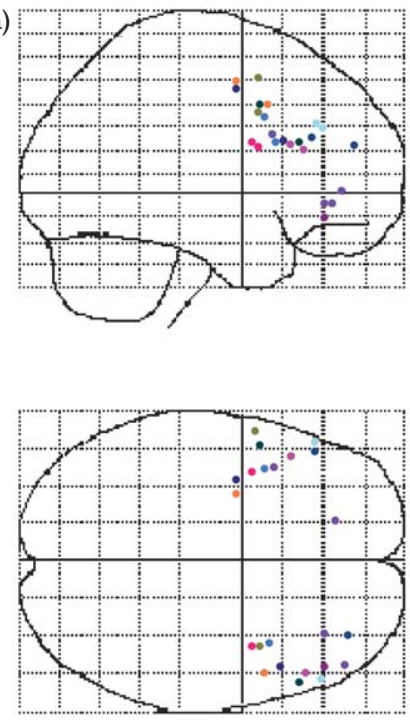

(b)

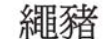

(d) 豬繩 (c)

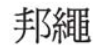

(e)

$\square$ ॐ $\Omega$

Fig. I (a) The bilaterally activated loci in the middle frontal gyrus in the standard Talairach space from the neuroimaging literature on Chinese characters. Different colors represent loci reported in different studies (see Table I, $[6,7,8,9,10, \mid 1,12,13,14,15])$. (b-e) Examples of stimuli used in this study (targets on the left, non-targets on the right). (b) The or thographic task, the left character contains the target radical 䋆. (c) The phonological task, the left character is pronounced as /bang/; the right one is pronounced as $/$ sheng/. (d) The semantic task, the left character means a pig; the right one means a rope. (e) The control task, the figure at left contains a rectangle.

mapping of orthography-to-semantics $[6,9,13]$. To test the hypotheses, we designed three tasks that demanded participants to rely predominantly on orthographic, phonological, or semantic processing to accomplish the task. The tasks and the stimuli were arranged to be comparable under the three conditions such that any activation contrast, if observed, could be attributed to differential processing of the character instead of other irrelevant variation in the tasks. As all characters were visually presented, processing of orthographic features was a prerequisite; thus, the right middle frontal gyrus should be commonly activated. In view of the evidence that a fluent reader will automatically or unintentionally assimilate phonological and/or semantic information as he sees a word [2,3], some activity in the left

middle frontal gyrus would also be observed during all character tasks, yet the activation should be differential and smaller for the orthographic task because phonological and semantic processes are subsidiary and not needed to correctly perform the task.

\section{Materials and methods \\ Participants}

Twelve healthy, right-handed, native Chinese speakers (six men, with ages ranging from 19 to 29 years) participated in this study. Informed consent was obtained from all volunteers. The experimental protocol was approved by the Human Subject Committee of the Medical School of National Taiwan University.

\section{Materials and procedures}

We selected three sets of stimuli for the orthographic, phonological, and semantic decision tasks, respectively, from the Academia Sinica Balanced Corpus [17], which contained 5656 Chinese characters ranked according to their linguistic frequencies. All the stimulus characters were selected from rank 1000 to 3000, and with a left-right configuration; that is, comprising two orthographic components arranged side by side (Fig. 1b-d). For each experimental condition, 18 characters, with frequencies matched across the sets, were chosen as targets. The targets of one condition were used as non-targets in the other two conditions. To prevent participants from detecting the purposes of stimulus design, an additional 18 characters were selected for each condition, and served as non-targets as well. Thus, each experimental stimulus set had 72 characters (18 targets and 54 non-targets). We also created 72 stimuli for the control condition to simulate the characters used in the experimental conditions (Fig. 1e); they were figures consisting of two simple geometric forms, arranged in a left-right configuration.

All participants performed three character decision tasks and a control task. In the orthographic condition, participants were instructed to respond by squeezing an airpressure response bulb with their right hands when the presented character contained the radical 後 (Fig. 1b). In the phonological condition, they were to respond if the pronunciation of a character started with a consonant /b/ (Fig. 1c). In the semantic condition, they were instructed to respond to characters that stood for animal names (Fig. 1d). 
Table 2 Talairach coordinates of the significantly activated brain regions for different contrasts

\begin{tabular}{|c|c|c|c|c|c|c|c|c|c|c|}
\hline \multirow[b]{2}{*}{ Brain region } & \multicolumn{5}{|c|}{ Left hemisphere } & \multicolumn{5}{|c|}{ Right hemisphere } \\
\hline & BA & $x$ & $y$ & $z$ & $t$ & BA & $x$ & $y$ & $z$ & $t$ \\
\hline \multicolumn{11}{|l|}{ Character $>$ control } \\
\hline \multirow[t]{4}{*}{ Middle frontal gyrus } & 46 & -45 & 33 & 15 & 3.41 & 46 & 51 & 30 & 21 & 4.78 \\
\hline & 46 & -48 & 27 & 24 & 3.17 & 9 & 45 & 15 & 30 & 4.42 \\
\hline & 9 & -42 & 12 & 27 & 3.25 & 9 & 54 & 18 & 30 & 3.41 \\
\hline & 46 & -45 & 36 & 24 & 3.26 & & & & & \\
\hline Middle temporal gyrus & 39 & -33 & -60 & 27 & 2.62 & & & & & \\
\hline Precuneus & & & & & & 39 & 33 & -66 & 30 & 3.19 \\
\hline Superior occipital gyrus & 19 & -33 & -72 & 30 & 2.69 & & & & & \\
\hline Fusiform gyrus & & & & & & 18 & 27 & -90 & -15 & 3.68 \\
\hline Character $<$ control & & & & & $\mathrm{N} / \mathrm{A}$ & & & & & \\
\hline \multicolumn{11}{|l|}{ Phonological $>$ orthographic } \\
\hline Superior frontal gyrus & & & & & & 6 & 3 & 18 & 54 & 2.86 \\
\hline Middle frontal gyrus & 47 & -39 & 39 & -3 & 3.10 & & & & & \\
\hline \multicolumn{11}{|l|}{ Semantic $>$ orthographic } \\
\hline Middle frontal gyrus & 47 & -45 & 36 & -3 & 2.85 & & & & & \\
\hline
\end{tabular}

BA, Brodmann area.

In the control condition, they made a response whenever a rectangle was included in a stimulus (Fig. 1e). Each of the three experimental conditions alternated with the control condition repeatedly for six times in a block-design run. The order of the runs was randomly determined. Each block consisted of 12 trials, each lasting for $1.75 \mathrm{~s}$. The stimulus presented in a trial was randomly selected from the corresponding stimulus set and presented through magnetic resonance imaging-compatible goggles (Visua Stim XGA, Resonance Technology Inc., Northridge, California, USA). The size of each stimulus was $1.7 \times 1.7^{\circ}$. All participants' responses were recorded and correct rates were higher than $99 \%$.

\section{Imaging procedure}

The images were acquired with a Bruker 30/90 Medspec 3-T scanner (Bruker BioSpin MRI GmbH, Ettlingen, Germany) using a standard birdcage head coil. Both functional (blood oxygenation level-dependent) and anatomical (T1weighted, $256 \times 256)$ images were obtained in identical planes. Twenty 5-mm transverse planes parallel to the anterior commissure-posterior commissure line were collected to cover the whole brain. A gradient-echo echo-planar imaging sequence was used to acquire the functional data $\left(\mathrm{TR}=3500 \mathrm{~ms}, \mathrm{TE}=35 \mathrm{~ms}\right.$, flip angle $=90^{\circ}$, matrix $=128 \times 128$, resolution $=2.34 \times 2.34 \mathrm{~mm}^{2}$ ). Each run lasted $262.5 \mathrm{~s}$ (75 images), and the first three images were excluded from the analyses.

\section{Data analysis}

The functional images were realigned, coregistered, smoothed, and normalized using SPM99 [18]. The images were smoothed by an isotropic Gaussian kernel with fullwidth at half-maximum of $6 \mathrm{~mm}$. We calculated the activation with a fixed-effect model by contrasting images acquired during an experimental condition with those acquired during the control condition using a voxel-specific $t$-test of SPM99. The contrasts between two of the experimental conditions were also examined ( $t$-value threshold=2.58, $P<0.005$, uncorrected). Then, a conjunction analysis was performed [19] to derive common activation patterns among the experimental conditions ( $t$-value threshold $=2.46, P<0.01$, corrected)

\section{Results}

Conjunction analysis across the three character conditions versus the control condition

Conjunction analysis was developed for statistically addressing the functional commonalities among the tasks across all participants by jointly rejecting the null hypotheses that aim to test for activation for each participant individually [19]. Figure 2a shows the common activation patterns among the orthographic, phonological, and semantic tasks versus the control task over the 12 participants. Table 2 shows the Talairach coordinates of those maximally activated voxels in the activated areas. The commonly activated areas are the middle frontal gyri [Brodmann area (BA) 46, 9] in both hemispheres; the precuneus (BA 39) and fusiform gyrus (BA 18) in the right hemisphere; and the middle temporal gyrus (BA 39) and superior occipital gyrus (BA 19) in the left hemisphere. Conversely, no common activation was found when comparing the control task with the three character tasks.

\section{Comparison of activation among the orthographic, phonological, and semantic tasks}

Compared with the orthographic task, the phonological task showed significant activation in the right superior frontal gyrus (BA 6) and left middle frontal gyrus (BA 47) (Fig. 2b, Table 2), and the semantic task only activated the left middle frontal gyrus (BA 47) (Fig. 2c, Table 2). We did not find significant cerebral activation by comparison between the phonological and semantic tasks.

\section{Discussion}

We investigated the functions subserved by the left and right middle frontal gyri by examining the neural activation accompanying the orthographic, phonological, and semantic processing of Chinese characters. Our results showed that the middle frontal gyrus of both hemispheres (BA 9, 46) contributes to character recognition. All character tasks 


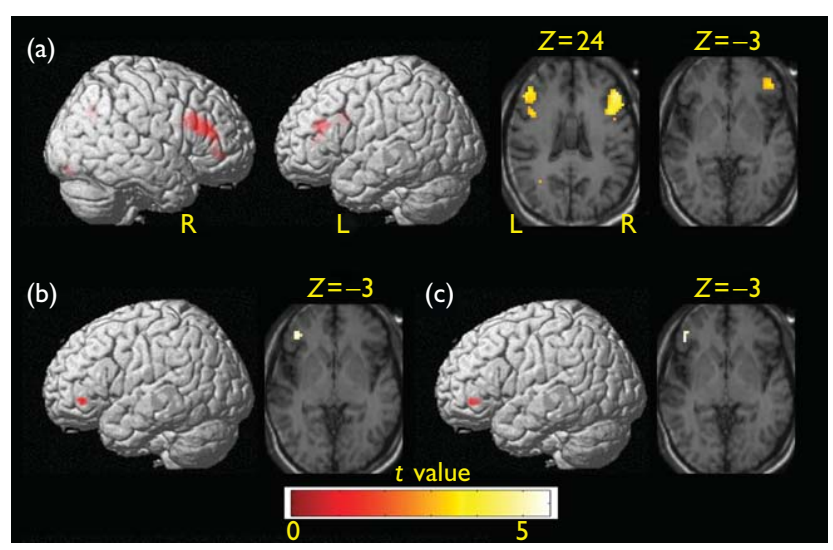

Fig. 2 Brain activation maps. (a) The common activation among the character conditions contrasted with the control condition. The axial view shows bilateral activation in the middle frontal gyrus overlaid on anatomical images. The pseudo-colored pixels denote the brain areas showing statistical significance. (b) Phonological task $>$ orthographic task, the axial view shows activation in the left middle frontal gyrus. (c) Semantic task $>$ orthographic task, the axial view shows activation in the left middle frontal gyrus, too.

demand similar orthographic analysis and we observed comparable activation in the right middle frontal gyrus. On the other hand, both the phonological and semantic tasks caused larger activation in the left middle frontal gyrus than the orthographic task. These data support our hypothesis.

We designed the control stimuli and task to simulate that of the orthographic task and attributed the differential activation to orthographic processing of characters. Illegal or nonsense characters are often used as control stimuli to probe how abstract configuration rules of characters may affect word recognition. Yet, the present study aimed to investigate the general orthography of characters, including strokes, radicals, and other relevant rules. Geometric forms may serve as a better control because they are devoid of any orthographical cues. On the basis of the task demands, we might intuitively assume that orthographic processing, but not the other two types of processing, is engaged in the orthographic task, and so are the cases for phonological and semantic tasks. Behavioral evidence, however, has rejected this idea $[2,3,20]$. For example, the well-known Stroop effect demonstrates how strongly the task-irrelevant semantics of a word can be automatically triggered to interfere with naming the printed color of the word [21]. Our results showing that the orthographic task activated the left middle frontal gyrus provide physiological evidence to support such automatic engagement and are consistent with the notion that the left middle frontal gyrus mediates the conversion of orthography to phonology and mapping of orthography to semantics $[6,9,13]$. In addition, the phonological and semantic tasks required extensive processing of phonology and semantics of a character, as compared with the orthographic task; hence, we detected greater activation in the left middle frontal gyrus, which lends further support to our hypothesis.

Studies on alphabetic languages failed to observe activation in the right middle frontal gyrus in tasks demanding an orthographic process [22,23]. Instead, the fusiform and lingual gyri have been proposed to process visual word forms in alphabetic languages [23] and, in particular, the left fusiform gyrus has been suggested as a visual word form area [24]. Our results detected activation in the right fusiform gyrus across all the character tasks (Fig. 2a, Table 2 ). Owing to the imbalance in visual complexities between the Chinese characters and geometric forms used as control stimuli in the present study, however, the right fusiform gyrus activation could be caused by processing general visual form rather than by the orthography per se.

We do not anticipate that character frequency would have influenced our results of bilateral activation of the middle frontal gyrus although only high-frequency characters were used. Table 1 indicated that the activation of both gyri is robust given that different frequency characters were compared [10,11]. It may be argued that different neural activity in the left middle frontal gyrus was due to different cognitive control or level of processing for tasks. It could not, however, explain why the same effect did not appear in comparison between the phonological and semantic tasks in which cognitive control for alternation of tasks remained. In addition, the semantic task presumably requires a deeper level of processing than the phonological one [25], and thus it should have generated the strongest activation among the three tasks. The failure of observing such an order renders the level of processing interpretation less likely.

\section{Conclusion}

By examining brain activation patterns during the performance of orthographic, phonological, and semantic tasks, the present study shows the functional dissociation between the left and the right middle frontal gyri when reading Chinese characters.

\section{Acknowledgements}

We would like to thank colleagues in the MRI Lab and Ms M.C. Chen for data collection and technical support.

\section{References}

1. Van Orden GC, Goldinger SD. Interdependence of form and function in cognitive systems explains perception of printed words. Special section: modeling visual word recognition. J Exp Psychol Hum Percept Perform 1994; 20:1269-1291.

2. Brysbaert M, Praet C. Reading isolated words: no evidence for automatic incorporation of the phonetic code. Psychol Res 1992; 54:91-102.

3. Perfetti CA, Bell L, Delaney S. Automatic phonetic activation in silent word reading: evidence from backward masking. J Mem Lang 1988; 27: 59-70.

4. Binder JR, Westbury CF, Mckiernan KA, Possing ET, Medler DA. Distinct brain systems for processing concrete and abstract concepts. J Cogn Neurosci 2005; 14:1088-1098.

5. Yoon HW, Cho KD, Chung JY, Park HW. Neural mechanisms of Korean word reading: a functional magnetic resonance imaging study. Neurosci Lett 2005; 373:206-211.

6. Siok WT, Perfetti CA, Jin Z, Tan LH. Biological abnormality of impaired reading is constrained by culture. Nature 2004; 431:71-76.

7. Fu SF, Chen Y, Smith S, Iversen S, Matthews PM. Effects of word form on brain processing of written Chinese. Neuroimage 2002; 17:1538-1548.

8. Li G, Cheung RT, Gao JH, Lee TM, Tan LH, Fox PT, et al. Cognitive processing in Chinese literate and illiterate subjects: an fMRI study. Hum Brain Mapp 2006; 27:144-152.

9. Tan LH, Feng CM, Fox PT, Gao JH. An fMRI study with written Chinese. Neuroreport 2001; 12:83-88.

10. Kuo WJ, Yeh TC, Lee CY, Wu YT, Chou CC, Ho LT, et al. Frequency effects of Chinese character processing in the brain: an event-related fMRI study. Neuroimage 2003; 18:720-730. 
11. Lee CY, Tsai JL, Kuo WJ, Yeh TC, Wu YT, Ho LT, et al. Neuronal correlates of consistency and frequency effects on Chinese character naming: an event-related fMRI study. Neuroimage 2004; 23:1235-1245.

12. Tan LH, Spinks JA, Gao JH, Liu HL, Perfetti CA, Xiong J, et al. Brain activation in the processing of Chinese characters and words: a functional MRI study. Hum Brain Mapp 2000; 10:16-27.

13. Tan LH, Liu HL, Perfetti CA, Spinks JA, Fox PT, Gao JH. The neural system underlying Chinese logograph reading. Neuroimage 2001; 13: 836-846.

14. Dong Y, Nakamura K, Okada T, Hanakawa T, Fukuyama H, Mazziotta JC, et al. Neural mechanisms underlying the processing of Chinese words: an fMRI study. Neurosci Res 2005; 52:139-145.

15. Kuo WJ, Yeh TC, Lee JR, Chen LF, Lee PL, Chen SS, et al. Frequency effects of Chinese character processing in the brain: an event-related fMRI study. Neuroimage 2004; 18:720-730.

16. Belger A, Puce A, Krystal JH, Gore JC, Goldman-Rakic PS, McCarthy G. Dissociation of mnemonic and perceptual processes during spatial and nonspatial working memory using fMRI. Hum Brain Mapp 1998; 6:14-32.

17. Academia Sinica balanced corpus (version 3) (1998). [CDROM]. Academia Sinica, Taipei, Taiwan.
18. Friston KJ, Holmes AP, Worsley KJ, Poline JP, Frith CD, Frackowiak RSJ. Statistical parametric maps in functional imaging: a general linear approach. Hum Brain Mapp 1995; 2:189-210.

19. Friston KJ, Holmes AP, Price CJ, Buchel C, Worsley KJ. Multisubject fMRI studies and conjunction analyses. Neuroimage 1999; 10:385-396.

20. Perfetti CA, Zhang S. Very early phonological activation in Chinese reading. J Exp Psychol Learn Mem Cogn 1995; 21:24-33.

21. Stroop JR. Studies of interference in serial verbal reactions. J Exp Psychol 1935; 18:643-662.

22. Price CJ. The anatomy of language: contributions from functional neuroimaging. J Anat 2000; 197:335-359.

23. Binder JR. Functional MRI of the language system. In: Moonen CTW Bandettini PA, editors. Functional MRI. New York: Springer; 1999. pp. 407-419.

24. Cohen L, Dehaene S, Naccache L, Lehericy S, Dehaene-Lambertz G, Henaff MA, et al. The visual word form area: spatial and temporal characterization of an initial stage of reading in normal subjects and posterior split-brain patients. Brain 2000; 123:291-307.

25. Craik FIM, Lockhart RS. Level of processing: a framework for memory research. J Verbal Learn Verbal Behav 1972; 11:671-684. 